\title{
Socioeconomic Inequalities in Frailty among Older Adults: Results from a 10-Year Longitudinal Study in the Netherlands
}

\author{
Emiel O. Hoogendijk ${ }^{a}$ Martijn W. Heymans ${ }^{\text {a }}$ Dorly J.H. Deeg ${ }^{\text {a }}$ \\ Martijn Huisman ${ }^{\mathrm{a}, \mathrm{b}}$ \\ ${ }^{a}$ Department of Epidemiology and Biostatistics, Amsterdam Public Health Research Institute, VU University Medical \\ Center, and ${ }^{\mathrm{b}}$ Department of Sociology, VU University, Amsterdam, The Netherlands
}

\section{Keywords}

Education - Epidemiology of aging · Frail elderly ·

Socioeconomic inequalities

\begin{abstract}
Background: Frailty is an important risk factor for adverse outcomes in older people. Substantial variation in frailty prevalence between socioeconomic groups exists, but longitudinal evidence for the association between socioeconomic position (SEP) and frailty is scarce. Objective: To investigate the course of socioeconomic inequalities in frailty among older adults during 10 years of follow-up. Methods: Data were used from the Longitudinal Aging Study Amsterdam $(n=1,509)$. Frailty was measured with the functional domains approach, based on deficiencies in four domains: physical, nutritive, cognitive, and sensory. Mixed-model analyses were performed to estimate the course of frailty and its association with SEP during a 10-year follow-up. We investigated whether similar patterns of associations held in different scenarios, comparing results of survivor analyses with those based on two imputation methods accounting for dropout due to death (substitution of first missing value and missing values imputed with a prediction model). $\boldsymbol{R e}$ sults: All scenarios showed a linear increase in frailty with
\end{abstract}

\section{KARGER}

E-Mail karger@karger.com www.karger.com/ger
(C) 2017 The Author(s)

Published by S. Karger AG, Basel This article is licensed under the Creative Commons Attribution-
NonCommercial-NoDerivatives 4.0 International License (CC BYNC-ND) (http://www.karger.com/Services/OpenAccessLicense). Usage and distribution for commercial purposes as well as any distribution of modified material requires written permission. aging (survivor analyses $\mathrm{OR}=1.87,95 \% \mathrm{Cl}=1.66-2.11$ ) and associations of low education and low income with frailty (adjusted OR for low education $=1.76,95 \% \mathrm{Cl}=1.05-2.97$; adjusted $\mathrm{OR}$ for low income $=1.90,95 \% \mathrm{Cl}=1.20-3.01$; both for survivor analyses). Sex-stratified analyses indicated that socioeconomic inequalities were mainly present in men, not in women. Similar patterns of associations of SEP with frailty were observed in all scenarios, but the increase in frailty prevalence over time differed substantially between the scenarios. There were no statistically significant interactions between time and SEP on frailty (all scenarios), suggesting that inequalities in frailty did not increase or decrease during follow-up. Conclusion: SEP inequalities in frailty among older adults were observed, mainly among men, and persisted during 10 years of follow-up.

(C) 2017 The Author(s)

Published by S. Karger AG, Basel

\section{Introduction}

Older men and women who are frail have higher chances of dying, being institutionalized, and having a decline in functioning than those who are not [1-3]. Frailty represents a lack of reserve capacity in one or more functional domains which makes people vulnerable to all

Emiel O. Hoogendijk, PhD

Department of Epidemiology and Biostatistics Amsterdam Public Health Research Institute, VU University Medical Center P.O. Box 7057, NL-1007 MB Amsterdam (The Netherlands)

E-Mail e.hoogendijk@vumc.nl 
kind of internal and environmental stressors [4]. Even though there are many operational definitions of frailty [5], it is generally agreed that frailty is a relevant geriatric condition that captures elements of physical and cognitive functioning that other known measures of morbidity, including measures of functional limitations or disability, do not [6].

Many frailty measures encompass low physical activity, weight loss or underweight, weakness (e.g., in arms or legs), and often also either slowness, problems with memory and attention, or reduced vision or hearing $[5,7,8]$. Frailty has clinical implications because it often constitutes a state of health that is still reversible or modifiable, but is close on the threshold to chronic irreversible and unmanageable health problems [4]. It has public health implications as well. In societies where older adults continue to live longer with chronic diseases, among other things due to improved treatment of disease, frailty becomes a key factor in identifying high-risk groups [9]. Variations in frailty between subgroups pinpoint opportunities for prevention of further health inequalities.

Studies suggest that a high socioeconomic position (SEP) protects against becoming frail [7, 10-15]. However, there is a lack of knowledge on the longitudinal relationship between these factors over an extended time period. Such studies may indicate whether the development of frailty in lower socioeconomic groups will be quicker than that in higher socioeconomic groups throughout later life. So far, two studies have pointed into the direction of persisting (not widening) SEP inequalities in frailty in old age $[16,17]$.

The aim of this study was to investigate the course of socioeconomic inequalities in frailty among older adults during 10 years of follow-up. Such an assessment is challenging because longitudinal studies of aging are hampered by rates of attrition due to death and health-related dropout, and they end up with selective samples of healthy participants. Previous studies did not take into account this potential selection bias. Therefore, we investigated whether similar patterns of associations held in different scenarios, comparing the results of survivor analyses with two imputation methods accounting for dropout due to death.

\section{Methods}

Sample

Data from the Longitudinal Aging Study Amsterdam (LASA) were used. Details on the sampling and data collection of LASA have been published before $[18,19]$. In short, LASA is a nationally representative study on physical, emotional, cognitive, and social functioning of older adults in the Netherlands, which started in 1992 and is still ongoing. LASA data are collected by trained interviewers in a face-to-face main interview and in a separate medical interview (including clinical tests) in the home of the respondents.

For this study, the second measurement wave of LASA (1995/ 1996) was chosen as our baseline measurement because key information on frailty markers was collected at that wave for the first time in LASA. Data from four measurement waves were included in this study (1995/1996, 1998/1999, 2001/2002, and 2005/2006), together covering 10 years of observation. We selected all participants with data from the medical interview in 1995/1996 who were 65 years or older at that time $(n=1,509)$. Of those, 710 were still alive after 10 years of follow-up and participated in the fifth LASA measurement wave (2005/2006); 696 (46.2\%) died during followup, and 102 dropped out because of other reasons (43 ineligible, 45 refused, 14 could not be contacted anymore). Vital status and date of death of participants were traced through municipal registries. Ascertainment was $100 \%$ complete.

\section{Independent Variables}

Our measures of SEP were educational level and household income category, both measured at baseline. Three categories of education were constructed: (1) low education included those who had primary education completed or less, (2) high education consisted of those who completed some form of tertiary education (such as university degrees or higher vocational education), and (3) those with some form of secondary education completed constituted the medium education group. Income was measured with a categorical question on monthly net household income at baseline. The household income of respondents living with a partner in the household was multiplied by 0.7 to make it comparable to the income of a one-person household. Income categories were collapsed into three overall categories corresponding with tertiles as closely as possible ( $37.4 \%$ in the lowest income category and $29.2 \%$ in the highest).

\section{Dependent Variables}

Our frailty measure followed the functional domains approach to frailty [7]. This approach views frailty as a grouping of problems and losses of capability. These problems and losses are postulated to be multisystemic and encompass deficiencies in four domains: physical, nutritive, cognitive, and sensory. We used low physical activity and low grip strength (physical domain), low body mass index (BMI) (nutritive domain), low cognitive functioning (cognitive domain), and vision and hearing problems (sensory domain).

To assess physical activity, respondents were asked how often and for how long they had walked, cycled, performed household activities, and played sports during the 2 weeks before the interview [20]. Respondents in the lowest quintile of total time spent on physical activity were assigned a value of 1 on this marker (all others a value of 0 ). Grip strength was measured with hand-held calibrated dynamometers. The maximum values of the left and right hands were summed. Respondents with grip strength levels in the lowest sex-specific quintile were assigned a value of 1 . BMI was calculated from height and weight measurements. Height was measured using a stadiometer and weight was measured with a calibrated bathroom scale. The cutoff for this frailty marker was at $\mathrm{BMI}<23$, as a $\mathrm{BMI}<23$ is associated with elevated mortality rates 
in old age [21]. Cognitive functioning was measured with the Mini-Mental State Examination (range 0-30, higher scores indicating better functioning) [22]. A score $<24$ points is commonly used to indicate low cognitive functioning, and we used this as the cutoff to determine who was frail on this frailty marker. Poor vision and hearing were defined as not being able to recognize someone's face at a distance of $4 \mathrm{~m}$ (with glasses or contact lenses if needed), and not being able to follow a conversation in a group of four people (with hearing aids if necessary) [23]. All frailty markers were measured the same way in each of the measurement waves. Respondents were considered frail if they had frailty markers present in two or more domains [7].

\section{Data Analyses}

We performed a series of mixed-model analyses to estimate the association of SEP with frailty and changes in frailty, and to account for attrition due to mortality. Ignoring information from those who died during follow-up leads to biased estimates of longitudinal relationships of predictors with health because they only apply to those who survived until the end of the study. Such findings can, for example, not be used to predict longitudinal associations in future generations of older adults, which are likely to have lower mortality rates than current generations of older adults [24]. When examining trajectories, excluding those who died from analysis yields biased estimates that favor the groups with the most deaths [25]. In our case, this means that the survivor analyses might underestimate the deleterious effect of a low SEP on frailty.

Our first analyses were linear mixed models on the group of survivors, i.e., performing a complete case analysis based on all available observations (participants are included until they drop out from the study). In a second set of analyses, we transformed our measure of frailty to incorporate estimates for those who died during follow-up, as proposed by Diehr et al. [24]. We transformed the values for the first missing value after death (and only the first) of the participant into a value of 1 , indicating frailty. This scenario was chosen to reflect a situation of increases in life expectancy (i.e., reductions in mortality rates in old age), coupled with increases in frailty, which is a likely scenario for future groups of Dutch older adults. Results on the basis of this scenario might more accurately show how socioeconomic inequalities would be affected if all those who died were to live somewhat longer (i.e., in our scenario roughly 3 years, corresponding to one extra measurement wave), but were to live in frailer health.

In a third set of analyses, we tested a more theoretical scenario to see how socioeconomic inequalities in frailty would develop if dropout due to mortality did not occur. Selective dropout due to mortality is often seen as an explanation for decreasing health inequalities in later life. This scenario gives an indication of the impact of selective dropout due to mortality on the magnitude of socioeconomic inequalities in frailty among older adults. We substituted all missing values of frailty due to death on the basis of a prediction model of frailty at baseline for frailty at later measurement waves $[24,26]$. This was done as follows. First, a continuous frailty score was calculated at baseline (T1), based on the number of frailty markers present (range $0-6$ ). Then, predicted probabilities for frailty at each follow-up measurement wave (T2, T3, and T4), corresponding to each value of the continuous frailty score at $\mathrm{T} 1$, were derived from logistic regression analyses using all available (nonimputed) data. For each follow-up measurement wave
(T2, T3, T4), a logistic regression analysis was done with a continuous frailty score at baseline (T1) as predictor and frailty (yes/ no) at the specific measurement wave as outcome. Then, we substituted missing values at T2, T3, and/or T4 due to death by either "frail" or "not frail" on the basis of the predictive probabilities corresponding with the frailty score at T1. Participants who died during follow-up and had a frailty score at $\mathrm{T} 1$ that predicted frailty at T2, T3, and/or T4 with a probability $\geq 0.5$ were coded as "frail" on those waves where data was missing. For others who died and had frailty scores at T1 that predicted frailty on subsequent measurement waves with a probability $<0.5$, the missing values were coded as "not frail."

In all sets of longitudinal models, we firstly tested associations of time (measurement wave) with frailty, adjusting for sex and year of birth. Secondly, we included level of education and partner status (yes/no) into the models to estimate the association of education with frailty, adjusted for partner status, sex, year of birth, and time. Since income could be a mediator of the association of education with frailty, income was included in a third model that included all variables from the previous models. Finally, we included interactions of SEP (education and income) with time to test whether associations of SEP with frailty became larger or smaller during follow-up. Given that SEP distributions may differ by sex and because of the fact that the risk for frailty is higher in females [4], we also performed sex-stratified analyses for all longitudinal models.

All analyses were done using SAS 9.2 (GLIMMIX procedure). In these generalized linear mixed models, the dependency in frailty measures within the same person due to the repeated measurement waves was accounted for by allowing the regression coefficients to differ between subjects (random effects). The number of repeated observations per person was also allowed to vary. The data were defined as follows: level 2 as participant and level 1 as repeated measurement occasions. It was investigated whether random slopes should be included in the models, but it appeared that they were not necessary to optimize the model fit. The time variable was linearly related to frailty in all models. We tested quadratic functions, but these did not improve the model fit.

\section{Results}

The baseline characteristics and prevalence of frailty in the subgroups across measurement waves are presented in Table 1. Prevalence of frailty was generally higher in older birth cohorts, in women, in participants with lower levels of education and lower incomes, and in participants without a partner.

For each measurement wave, the prevalence of frailty and the six frailty markers are listed in Table 2. Low grip strength appeared to be the most common marker of frailty, together with low physical activity, whereas poor vision was the least common. Since this sample changed between measurement waves due to attrition, changes in percentages between measurement waves cannot be interpreted as trajectories. 
Table 1. Baseline characteristics and prevalence of frailty by characteristics at each measurement wave (survivor sample)

\begin{tabular}{|c|c|c|c|c|c|}
\hline & \multirow{2}{*}{$\frac{\text { Baseline, total }}{1995 / 1996}$} & \multicolumn{4}{|c|}{ Frailty by wave } \\
\hline & & $1995 / 1996$ & 1998/1999 & $2001 / 2002$ & $2005 / 2006$ \\
\hline \multicolumn{6}{|l|}{ Birth cohort } \\
\hline $1928-1932$ & $272(18.0)$ & $17(6.8)$ & $16(7.5)$ & $14(7.7)$ & $23(14.1)$ \\
\hline $1923-1927$ & $316(20.9)$ & $41(11.4)$ & $41(13.3)$ & $52(20.2)$ & $53(27.2)$ \\
\hline $1918-1922$ & $311(20.6)$ & $53(17.0)$ & $56(23.3)$ & $40(24.0)$ & $37(35.6)$ \\
\hline $1913-1917$ & $361(23.9)$ & $97(30.7)$ & $87(40.1)$ & $51(39.2)$ & $34(58.6)$ \\
\hline $1908-1912$ & $249(16.5)$ & $135(49.8)$ & $69(51.5)$ & $32(55.2)$ & $9(52.9)$ \\
\hline Men & $728(48.2)$ & $135(18.6)$ & $100(19.3)$ & $63(17.6)$ & $45(20.3)$ \\
\hline Women & $781(51.8)$ & $208(26.6)$ & $169(28.4)$ & $126(28.9)$ & $111(35.2)$ \\
\hline Low education & $645(42.7)$ & $181(28.1)$ & $142(31.3)$ & $98(30.6)$ & $72(36.9)$ \\
\hline Medium education & $681(45.1)$ & $124(18.2)$ & $105(19.7)$ & $72(19.0)$ & $66(23.7)$ \\
\hline High education & $181(12.0)$ & $37(20.4)$ & $22(17.7)$ & $19(20.4)$ & $18(28.1)$ \\
\hline Lowest income tertile & $440(32.7)$ & $137(31.2)$ & $108(35.0)$ & $69(33.0)$ & $55(42.3)$ \\
\hline Middle income tertile & $401(29.8)$ & $78(19.5)$ & $70(23.9)$ & $44(20.8)$ & $40(28.2)$ \\
\hline Highest income tertile & $503(37.4)$ & $74(14.7)$ & $61(15.0)$ & $59(19.8)$ & $51(24.3)$ \\
\hline No partner & $541(35.9)$ & $182(33.6)$ & $142(37.8)$ & $94(39.2)$ & $61(42.4)$ \\
\hline Partner living in house & $908(60.3)$ & $150(16.5)$ & $115(16.7)$ & $88(16.9)$ & $94(25.3)$ \\
\hline Partner outside of household & $57(3.8)$ & $10(17.5)$ & $11(24.4)$ & $7(22.6)$ & $1(4.5)$ \\
\hline
\end{tabular}

Table 2. Percentages of frailty and frailty markers (survivor sample)

\begin{tabular}{|c|c|c|c|c|c|c|c|}
\hline & \multirow{2}{*}{$\frac{\text { Frailty }}{\text { frail, \% }}$} & \multicolumn{6}{|c|}{ Frailty markers } \\
\hline & & $\begin{array}{l}\text { low physical } \\
\text { activity, \% }\end{array}$ & $\begin{array}{l}\text { low grip } \\
\text { strength, \% }\end{array}$ & $\begin{array}{l}\text { low BMI, } \\
\%\end{array}$ & $\begin{array}{l}\text { low cognitive } \\
\text { functioning, } \%\end{array}$ & $\begin{array}{l}\text { poor } \\
\text { vision, \% }\end{array}$ & $\begin{array}{l}\text { poor } \\
\text { hearing, } \%\end{array}$ \\
\hline $1995 / 1996$ & 22.7 & 20.0 & 21.4 & 16.1 & 13.8 & 6.0 & 15.6 \\
\hline 1998/1999 & 24.2 & 18.9 & 30.4 & 12.6 & 11.9 & 5.8 & 15.8 \\
\hline $2001 / 2002$ & 23.8 & 20.6 & 28.8 & 13.9 & 12.3 & 6.3 & 16.7 \\
\hline $2005 / 2006$ & 29.0 & 27.4 & 29.1 & 14.3 & 14.4 & 6.1 & 19.6 \\
\hline
\end{tabular}

BMI, body mass index.

Across the study period of 10 years, the 1,509 participants provided on average 2.6 observations. Dropout due to mortality during follow-up according to the level of education was as follows: low education $(50.2 \%, n=324)$, medium education $(41.1 \%, n=280)$, and high education $(50.8 \%, n=93)$. Frailty prevalence at baseline differed between survivors and people who deceased during the 10year follow-up period (11.1 vs. $36.4 \%$ ).

Table 3 shows the results of the mixed-model analyses. There was a general tendency of increased prevalence of frailty over time. In all three datasets the prevalence of frailty increased linearly during follow-up (ORs $>1$ ), but the increase in risk of frailty was largest in the sample where the first missing value after death was set to frail $(\mathrm{OR}=3.18,95 \% \mathrm{CI}=2.82-3.58)$. The sex-stratified analyses showed a similar pattern in both men and women (Table 4).

Educational and income gradients in frailty were found. Survivor analyses demonstrated that a higher risk of frailty after adjustment for age, sex, and partner status 
Table 3. Mixed model analyses for the total study population: Associations of time and socioeconomic factors with frailty

\begin{tabular}{|c|c|c|c|}
\hline & $\begin{array}{l}\text { Model 1: time } \\
\text { adjusted for sex and } \\
\text { birth cohort }\end{array}$ & $\begin{array}{l}\text { Model } 2 \text { : model } 1+ \\
\text { education and } \\
\text { partner status }\end{array}$ & $\begin{array}{l}\text { Model 3: model } 2+ \\
\text { income }\end{array}$ \\
\hline \multicolumn{4}{|l|}{ Survivor sample ${ }^{a}$} \\
\hline High education & & 1.00 & 1.00 \\
\hline Medium education & & $1.03(0.62-1.73)$ & $0.85(0.49-1.47)$ \\
\hline Low education & & $1.76(1.05-2.97)$ & $1.30(0.73-2.31)$ \\
\hline Highest income tertile & & & 1.00 \\
\hline Middle income tertile & & & $1.16(0.76-1.77)$ \\
\hline Lowest income tertile & & & $1.90(1.20-3.01)$ \\
\hline Time (measurement wave) & $1.87(1.66-2.11)$ & $1.89(1.67-2.13)$ & $1.93(1.71-2.19)$ \\
\hline Respondents/observations, $n$ & $1,509 / 3,950$ & $1,504 / 3,941$ & $1,343 / 3,551$ \\
\hline \multicolumn{4}{|c|}{ Imputation 1: first missing substituted with "frail" } \\
\hline High education & & 1.00 & 1.00 \\
\hline Medium education & & $0.91(0.58-1.42)$ & $0.75(0.46-1.20)$ \\
\hline Low education & & $1.73(1.09-2.73)$ & $1.28(0.77-2.11)$ \\
\hline Highest income tertile & & & 1.00 \\
\hline Middle income tertile & & & $1.18(0.82-1.71)$ \\
\hline Lowest income tertile & & & $1.98(1.31-2.99)$ \\
\hline Time (measurement wave) & $3.18(2.82-3.58)$ & $3.19(2.83-3.59)$ & $3.24(2.86-3.67)$ \\
\hline Respondents/observations, $n$ & $1,509 / 4,690$ & $1,504 / 4,677$ & $1,343 / 4,209$ \\
\hline \multicolumn{4}{|c|}{ Imputation 2: missing values imputed with prediction model } \\
\hline High education & & 1.00 & 1.00 \\
\hline Medium education & & $1.00(0.54-1.85)$ & $0.79(0.42-1.49)$ \\
\hline Low education & & $1.76(0.95-3.26)$ & $1.18(0.61-2.31)$ \\
\hline Highest income tertile & & & 1.00 \\
\hline Middle income tertile & & & $1.17(0.72-1.93)$ \\
\hline Lowest income tertile & & & $2.28(1.33-3.90)$ \\
\hline Time (measurement wave) & $1.62(1.47-1.79)$ & $1.62(1.47-1.79)$ & $1.64(1.48-1.81)$ \\
\hline Respondents/observations, $n$ & $1,509 / 4,690$ & $1,504 / 4,677$ & $1,343 / 4,209$ \\
\hline
\end{tabular}

${ }^{a}$ Analyses with the survival sample are based on all available observations. Participants are included until they drop out from the study.

was associated with low SEP, i.e., the low education group $(\mathrm{OR}=1.76,95 \% \mathrm{CI}=1.05-2.97)$ and the lowest income group $(\mathrm{OR}=1.90,95 \% \mathrm{CI}=1.20-3.01)$. A considerable part of the low education OR was attenuated after including income in the model (low education $\mathrm{OR}=1.30,95 \%$ $\mathrm{CI}=0.73-2.31$ ), suggesting that income is in the pathway between education and frailty. The sex-stratified analyses showed that these results only pertain to men, not to women.

After adjustment for partner status and the alternative indicator of SEP, similar patterns of associations of SEP with frailty were observed in the two samples with imputed frailty scores as compared to the survivor sample, although the magnitude of point estimates varied (mod- els 2 and 3 in Table 3 and men in Table 4). Results from the two imputed samples confirmed that the men with lower education (but not those with medium education) had higher odds of frailty as compared to those with higher education. For women, no statistically significant associations between SEP and frailty were observed, except for low income in model 3 of imputation method 2 $(\mathrm{OR}=2.03,95 \% \mathrm{CI}=1.01-4.12)$.

Interaction effects of SEP with time on frailty were estimated for the total population and for men and women separately (results not shown). No statistically significant interaction effects were found, suggesting that the association between SEP and frailty did not increase or decrease over time. 
Table 4. Mixed model analyses stratified by sex: Associations of time and socioeconomic factors with frailty

\begin{tabular}{|c|c|c|c|c|c|c|}
\hline & \multicolumn{2}{|c|}{$\begin{array}{l}\text { Model 1: time adjusted for birth } \\
\text { cohort }\end{array}$} & \multicolumn{2}{|c|}{$\begin{array}{l}\text { Model 2: model } 1+\text { education and } \\
\text { partner status }\end{array}$} & \multicolumn{2}{|c|}{ Model 3: model $2+$ income } \\
\hline & men & women & men & women & men & women \\
\hline High education & & & 1.00 & 1.00 & 1.00 & 1.00 \\
\hline Medium education & & & $1.16(0.59-2.25)$ & $0.66(0.42-1.02)$ & $0.87(0.44-1.72)$ & $0.85(0.35-2.08)$ \\
\hline Low education & & & $2.36(1.16-4.83)$ & $0.72(0.33-1.57)$ & $1.64(0.77-3.50)$ & $1.12(0.46-2.73)$ \\
\hline Lowest income tertile & & & & & $2.52(1.28-4.99)$ & $1.67(0.89-3.15)$ \\
\hline Time (measurement wave) & $1.67(1.38-2.02)$ & $2.00(1.72-2.34)$ & $1.69(1.39-2.04)$ & $2.02(1.73-2.35)$ & $1.76(1.45-2.14)$ & $2.04(1.74-2.40)$ \\
\hline Respondents/observations, $n$ & $728 / 1,823$ & $781 / 2,127$ & $725 / 1,818$ & $779 / 2,123$ & $662 / 1,664$ & $681 / 1,887$ \\
\hline \multicolumn{7}{|c|}{ Imputation 1: first missing substituted with "frail" } \\
\hline High education & & & 1.00 & 1.00 & 1.00 & 1.00 \\
\hline Medium education & & & $0.83(0.47-1.47)$ & $0.62(0.42-0.94)$ & $0.67(0.37-1.20)$ & $0.89(0.39-2.04)$ \\
\hline Time (measurement wave) & $3.58(2.96-4.34)$ & $2.91(2.50-3.39)$ & $3.59(2.96-4.35)$ & $2.91(2.50-3.39)$ & $3.69(3.02-4.50)$ & $2.92(2.49-3.44)$ \\
\hline Respondents/observations, $n$ & $728 / 2,235$ & $781 / 2,455$ & $725 / 2,228$ & $779 / 2,449$ & $662 / 2,040$ & $681 / 2,169$ \\
\hline \multicolumn{7}{|c|}{ Imputation 2: missing values imputed with prediction model } \\
\hline High education & & & 1.00 & 1.00 & 1.00 & 1.00 \\
\hline Medium education & & & $1.44(1.23-1.67)$ & $0.62(0.38-1.02)$ & $0.91(0.39-2.15)$ & $0.66(0.25-1.78)$ \\
\hline Low education & & & $1.28(0.55-3.00)$ & $0.82(0.34-1.98)$ & $1.66(0.64-4.33)$ & $0.89(0.33-2.40)$ \\
\hline Highest income tertile & & & & & 1.00 & 1.00 \\
\hline Middle income tertile & & & & & $1.04(0.51-2.12)$ & $1.36(0.67-2.73)$ \\
\hline Lowest income tertile & & & & & $3.10(1.32-7.30)$ & $2.03(1.01-4.12)$ \\
\hline Time (measurement wave) & $1.43(1.23-1.67)$ & $1.75(1.54-2.00)$ & $1.44(1.23-1.67)$ & $1.76(1.54-2.00)$ & $1.48(1.27-1.74)$ & $1.74(1.52-2.00)$ \\
\hline Respondents/observations, $n$ & $728 / 2,235$ & $781 / 2,455$ & $725 / 2,228$ & $779 / 2,449$ & $662 / 2,040$ & $681 / 2,169$ \\
\hline
\end{tabular}

a Analyses with the survival sample are based on all available observations. Participants are included until they drop out from the study.

\section{Discussion}

In the current study, we investigated the longitudinal relationships of SEP with frailty during a 10-year follow-up among a sample of Dutch older adults. We investigated whether similar patterns in these relationships held in different scenarios, comparing survivor analyses with two imputation methods of accounting for dropout due to death. In each of these scenarios, a linear increase in frailty over time was found. However, we observed that the increase in the prevalence of frailty over time differed substantially between the different scenarios. The patterns of associations of SEP with frailty were remarkably similar in all three samples, pointing toward low SEP - low education and the lowest income tertile - as determinants of frailty among men. For women, no associations between SEP and frailty were observed, except for low income in one of the imputed samples.
Our results confirm the findings from previous crosssectional and longitudinal studies that socioeconomic factors such as educational level and income are associated with frailty in older adults $[11,13,16]$. Our sex-stratified analyses revealed that SEP inequalities in frailty were not present among women in most of the analytical models. This contradicts previous studies where associations between SEP and frailty were also found in women $[11,15]$. We were able to investigate whether SEP associations with frailty increased or decreased during a follow-up period of 10 years. In line with a previous study over an extended time period, we observed that SEP inequalities in frailty remained the same during follow-up [16].

We performed sensitivity analyses to account for attrition caused by death during follow-up. We compared results to see whether general conclusions about the pattern of the associations of SEP with frailty could be drawn. The design of different scenarios in this way can 
be informative, especially when researchers hold expectations about future developments in survival and rates of ill-health. We investigated what the pattern and magnitude of socioeconomic inequalities in frailty would be if all who died during follow-up were to live 3 years longer, but would do so in frail health (imputation 1). This scenario was designed in line with declines in mortality rates in Dutch older men and women and with findings from analyses on LASA data showing that younger cohorts of older men and women have a higher prevalence of chronic disease than older cohorts, suggesting that they will be frailer on average [27]. It would be of great value to investigate to what extent our scenario plays out in future cohorts in Dutch men and women who are expected to live longer, but will do so in worse health.

The other scenario that we investigated was more theoretical, to see what would happen with socioeconomic inequalities in frailty if there was no (selective) dropout due to mortality (imputation 2). Interestingly, there were no large differences between the survivor analyses and the analyses in this scenario. An explanation for this might be that there was no clear pattern of SEP-specific mortality in this study sample. Perhaps the largest part of selection has already taken place before the age of 65 . For this scenario, we chose a single imputation method, based on a validated procedure [26]. However, in future research other methods such as multiple imputation should be investigated, as well as other scenarios that test joint modelling of frailty and death [28].

An important limitation of the study may be that the LASA measurement waves were conducted at 3-year intervals. Participants who dropped out due to mortality may have experienced brief periods of rapid decline in functioning that do not show up in our data if they occurred between measurement waves. It is known that especially cognitive functioning often exhibits increased declines in proximity to death $[29,30]$. Another limita- tion is that our imputation methods negate the possibility that participants died because of the development of frailty between two measurement waves. We do not exactly know in how many cases frailty was the main cause of death.

In conclusion, our study provides robust evidence for the longitudinal association between SEP and frailty in Dutch older people, in particular among older men. Our sensitivity analyses showed that it is difficult to pinpoint exact estimations of the magnitude of inequalities if one wants to account for mortality-related attrition. In all scenarios, we observed SEP inequalities in frailty during follow-up to be persisting rather than widening. Since frailty represents an intermediate position between better health and more advanced health problems, institutionalization, and death [31], frailty should be considered a potential mediator of socioeconomic inequalities in these outcomes. Public health strategies aimed at reducing frailty may therefore be important to reduce health inequalities in later life.

\section{Acknowledgments}

The Longitudinal Aging Study Amsterdam is largely supported by a grant from the Netherlands Ministry of Health, Welfare, and Sports, Directorate of Long-Term Care.

\section{Statement of Ethics}

The LASA study was approved by the Medical Ethics Committee of the VU University Medical Center. Informed consent was obtained from all participants.

\section{Disclosure Statement}

The authors have no potential conflicts of interest to disclose.

\section{References}

1 Fried LP, Tangen CM, Walston J, Newman AB, Hirsch C, Gottdiener J, Seeman T, Tracy R, Kop WJ, Burke G, McBurnie MA: Frailty in older adults: evidence for a phenotype. J Gerontol A Biol Sci Med Sci 2001;56:M146M156.

2 Drubbel I, de Wit NJ, Bleijenberg N, Eijkemans RJ, Schuurmans MJ, Numans ME: Prediction of adverse health outcomes in older people using a frailty index based on routine primary care data. J Gerontol A Biol Sci Med Sci 2013;68:301-308.
3 Hoogendijk EO, Suanet B, Dent E, Deeg DJ, Aartsen MJ: Adverse effects of frailty on social functioning in older adults: results from the Longitudinal Aging Study Amsterdam. Maturitas 2016;83:45-50.

4 Clegg A, Young J, Iliffe S, Rikkert MO, Rockwood K: Frailty in elderly people. Lancet 2013; 381:752-762.

5 Dent E, Kowal P, Hoogendijk EO: Frailty measurement in research and clinical practice: a review. Eur J Intern Med 2016;31:3-10.
6 Morley JE, Vellas B, van Kan GA, Anker SD, Bauer JM, Bernabei R, Cesari M, Chumlea WC, Doehner W, Evans J, Fried LP, Guralnik JM, Katz PR, Malmstrom TK, McCarter RJ, Gutierrez Robledo LM, Rockwood K, von Haehling S, Vandewoude MF, Walston J: Frailty consensus: a call to action. J Am Med Dir Assoc 2013;14:392-397.

7 Strawbridge WJ, Shema SJ, Balfour JL, Higby HR, Kaplan GA: Antecedents of frailty over three decades in an older cohort. J Gerontol B Psychol Sci Soc Sci 1998;53:S9-S16. 
8 Sourial N, Bergman H, Karunananthan S, Wolfson C, Guralnik J, Payette H, GutierrezRobledo L, Deeg DJ, Fletcher JD, Puts MT, Zhu B, Beland F: Contribution of frailty markers in explaining differences among individuals in five samples of older persons. J Gerontol A Biol Sci Med Sci 2012;67:11971204.

9 Cesari M, Marzetti E, Thiem U, Perez-Zepeda MU, Abellan van Kan G, Landi F, Petrovic M, Cherubini A, Bernabei R: The geriatric management of frailty as paradigm of "The end of the disease era." Eur J Intern Med 2016;31: 11-14.

10 Syddall H, Roberts HC, Evandrou M, Cooper C, Bergman H, Aihie Sayer A: Prevalence and correlates of frailty among community-dwelling older men and women: findings from the Hertfordshire Cohort Study. Age Ageing 2010;39:197-203.

11 Szanton SL, Seplaki CL, Thorpe RJ Jr, Allen $\mathrm{JK}$, Fried LP: Socioeconomic status is associated with frailty: the Women's Health and Aging Studies. J Epidemiol Community Health 2010;64:63-67.

12 Woo J, Chan R, Leung J, Wong M: Relative contributions of geographic, socioeconomic, and lifestyle factors to quality of life, frailty, and mortality in elderly. PLoS One 2010;5: e8775.

13 Etman A, Burdorf A, van der Cammen TJ, Mackenbach JP, van Lenthe FJ: Socio-demographic determinants of worsening in frailty among community-dwelling older people in 11 European countries. J Epidemiol Community Health 2012;66:1116-1121.

14 Romero-Ortuno R: Frailty index in Europeans: association with determinants of health. Geriatr Gerontol Int 2014;14:420-429.

15 Gardiner PA, Mishra GD, Dobson AJ: The effect of socioeconomic status across adulthood on trajectories of frailty in older women. J Am Med Dir Assoc 2016;17:372.e1-e3.
16 Stolz E, Mayerl H, Waxenegger A, Rasky E, Freidl W: Impact of socioeconomic position on frailty trajectories in 10 European countries: evidence from the Survey of Health, Ageing and Retirement in Europe (20042013). J Epidemiol Community Health 2017; 71:73-80.

17 Hoogendijk EO, van Hout HP, Heymans MW, van der Horst HE, Frijters DH, Broese van Groenou MI, Deeg DJ, Huisman M: Explaining the association between educational level and frailty in older adults: results from a 13-year longitudinal study in the Netherlands. Ann Epidemiol 2014;24:538-544.e2.

18 Huisman M, Poppelaars J, van der Horst M, Beekman AT, Brug J, van Tilburg TG, Deeg DJ: Cohort profile: the Longitudinal Aging Study Amsterdam. Int J Epidemiol 2011;40: 868-876.

19 Hoogendijk EO, Deeg DJ, Poppelaars J, van der Horst M, Broese van Groenou MI, Comijs HC, Pasman HR, van Schoor NM, Suanet B, Thomese F, van Tilburg TG, Visser M, Huisman M: The Longitudinal Aging Study Amsterdam: cohort update 2016 and major findings. Eur J Epidemiol 2016;31:927-945.

20 Stel VS, Smit JH, Pluijm SM, Visser M, Deeg DJ, Lips P: Comparison of the LASA physical activity questionnaire with a 7-day diary and pedometer. J Clin Epidemiol 2004;57:252258.

21 Breeze E, Clarke R, Shipley MJ, Marmot MG, Fletcher AE: Cause-specific mortality in old age in relation to body mass index in middle age and in old age: follow-up of the Whitehall cohort of male civil servants. Int J Epidemiol 2006;35:169-178.
22 Folstein MF, Folstein SE, McHugh PR: "Minimental state." A practical method for grading the cognitive state of patients for the clinician. J Psychiatr Res 1975;12:189-198.

23 Statistics Netherlands: Health interview questionnaire. Heerlen, Statistics Netherlands, 1989.

24 Diehr P, Johnson LL, Patrick DL, Psaty B: Methods for incorporating death into healthrelated variables in longitudinal studies. J Clin Epidemiol 2005;58:1115-1124.

25 Diehr P, Johnson LL: Accounting for missing data in end-of-life research. J Palliat Med 2005;8(suppl 1):S50-S57.

26 Diehr P, Patrick DL, McDonell MB, Fihn SD: Accounting for deaths in longitudinal studies using the sf-36: the performance of the Physical Component Scale of the Short Form 36item health survey and the PCTD. Med Care 2003;41:1065-1073.

27 Deeg DJ, Huisman M: Cohort differences in 3 -year adaptation to health problems among Dutch middle-aged, 1992-1995 and 20022005. Eur J Ageing 2010;7:157-165.

28 MacDonald SW, Hultsch DF, Dixon RA: Aging and the shape of cognitive change before death: terminal decline or terminal drop? J Gerontol B Psychol Sci Soc Sci 2011;66:292301.

29 Kurland BF, Johnson LL, Egleston BL, Diehr $\mathrm{PH}$ : Longitudinal data with follow-up truncated by death: match the analysis method to research aims. Stat Sci 2009;24:211-227.

30 Bosworth HB, Siegler IC: Terminal change in cognitive function: an updated review of longitudinal studies. Exp Aging Res 2002;28: 299-315.

31 Cesari M, Prince M, Thiyagarajan JA, De Carvalho IA, Bernabei R, Chan P, Gutierrez-Robledo LM, Michel JP, Morley JE, Ong P, Rodriguez Manas L, Sinclair A, Won CW, Beard J, Vellas B: Frailty: An emerging public health priority. J Am Med Dir Assoc 2016;17:188192. 